\title{
CARTOGRAPHIE PAR TELEDETECTION DE L'EVOLUTION DES PLANTATIONS RESINEUSES DANS L'EST DE LA FORÊT BOREALE CANADIENNE
}

\author{
Osvaldo Valeria, Florence Lafon, Suzanne Brais et Ahmed Laamrani \\ Institut de recherche sur les forêts. Université du Québec en Abitibi Témiscamingue, \\ Rouyn Noranda (Québec) J9X 5E4, Canada
}

\begin{abstract}
Résumé
Au Québec, la remise en production par voie de plantation de résineux a débuté au début des années 1980 et atteint un sommet en 1990. Toutefois, on se demande si les efforts consacrés à la remise en production ont donné fruits compte tenu des problèmes liés à la compétition végétale affectant certaines plantations. Les objectifs du projet étaient de caractériser les changements de composition du couvert forestier de plantations résineuses établies depuis 1985 sur des sols argileux en milieu boréal. La caractérisation a été réalisée à l'aide d'indices de végétation extraits d'images satellitaires Landsat TM et ETM+ pour les périodes 2000, 2005 et 2010. Des orthophotographies numériques et des données terrain ont été utilisées afin de mesurer l'efficacité des indices. L'indice Tasseled Cap Greenness s'est avéré le plus performant à discriminer le type de couvert avec un indice de Kappa de $50 \%$. Les résultats montrent qu'en 200 plus de $50 \%$ des plantations étaient classifiées comme mixtes et $25 \%$ étaient classifiées comme feuillues, alors qu'en 2010 , $51 \%$ des plantations étaient mixtes (mélange feuillus - résineux) et $11 \%$ feuillues. À l'aide d'un modèle généralisé linéaire mixte avec approximation de Laplace nous avons mis en relation les changements de couvert et l'âge de la plantation. La probabilité pour un couvert initial feuillu d'évoluer vers un couvert mixte ou résineux est près de $68 \%$. Les variables type, âge et couvert de la plantation ainsi que leurs interactions ont des effets significatifs sur la probabilité de changement de couvert avec le temps. Ce projet a permis de faire le bilan de plantations résineuses établies entre 1980 et 1995 sur un territoire de 18797 ha et confirme que les outils de télédétection sont efficaces pour le suivi d'un grand territoire, en offrant des informations de qualité pour la planification des traitements sylvicoles.
\end{abstract}

Mots-clés : Probabilité de changement, Plantations, Résineux, Landsat ETM+

\begin{abstract}
In Quebec, reforestation by means of coniferous plantation began in the early 1980 and peaked in 1990. However, one wonders if these efforts bore fruits given the high degree of vegetation competition encountered in some plantations. The study objectives were to evaluate and compare changes in forest cover of coniferous plantations established since 1985 on clay soils in the boreal forest. The evaluation was conducted using vegetation indices extracted from images provided by Landsat TM and ETM + for the periods 2000, 2005 and 2010. Digital ortho-photos and field data were used to assess the effectiveness of these indices. The Tasseled Cap Greenness Index proved to be the most efficient to discriminate the type of cover with a Kappa index of $50 \%$. The results show that over $50 \%$ of the plantations were classified as mixed (deciduous - softwood) and $25 \%$ were classified as deciduous in 2000 , while in $2010,51 \%$ of plantations were mixed and $11 \%$ deciduous. Using a generalized linear mixed model with Laplace approximation, we have established the relationship between changes in cover, age and type of the plantation. The likelihood of an initial deciduous cover to evolve toward a mixed or coniferous cover was nearly $68 \%$. The plantation type, its age and cover and their interactions all had significant effects on the probability of a cover to change over time. This project contributed to the assessment of coniferous plantations established between 1980 and 1995 over an area of 18797 ha and confirms that remote sensing tools are effective for tracking large areas while providing quality information for the planning of silvicultural treatments.
\end{abstract}

Keywords: plantation, softwood, cover type detection, Landsat, cover change probability

\section{Introduction}

La forêt boréale est le deuxième plus grand biome terrestre et couvre la majorité de la partie nord de l'Eurasie et de l'Amérique. Au Canada, la forêt boréale occupe environ trois cent neuf millions d'hectares (Price et al., 2013), soit le tiers des forêts boréales encore existantes dans le monde. Au Québec, la forêt boréale est présente dans les régions de la Côte-Nord, le Saguenay-Lac-Saint-Jean, l'Abitibi-Témiscamingue ainsi que le Nord-du-Québec et s'étend sur différentes zones bioclimatiques : la sapinière à bouleau blanc, la pessière à lichen, la toundra forestière, et les pessières à mousse de l'Est et de l'Ouest. Les forêts nordiques résineuses occupent une grande partie de la forêt boréale en Amérique du Nord (Hollingsworth et al., 2006). Elles revêtent une importance économique majeure, tout en soutenant d'une manière très considérable l'industrie forestière. Pour assurer une pérennité de cette ressource, l'industrie forestière a souvent recours à du reboisement (plantations).
Dans les plantations de résineux sur les sites riches de la région de l'Abitibi à l'est du Canada, la compétition par les espèces arbustives, plus particulièrement les feuillus intolérants à l'ombre, est sévère (Thiffault et al., 2003). L'industrie forestière dans cette région priorise essentiellement les essences résineuses et les espèces feuillues sont peu prisées. Le contrôle mécanique de la compétition et l'éclaircie précommerciale dans ces plantations épargnent une composante plus ou moins importante de ces feuillus. La présence de ces dernières dans les plantations de conifères entraîne souvent une perte de rendement en raison de la concurrence accrue pour les ressources (Jobidon, 2000; Thiffault et al., 2003). Toutefois, un certain nombre d'auteurs ont suggéré que les peuplements ou plantations mélangés pourraient présenter une productivité plus élevée (Paré et Bergeron, 1995; Man et Lieffers, 1999; Loreau et al., 2001; Légaré et al., 2005) découlant d'une plus grande ségrégation des niches entre les conifères et les feuillus (Rothe et Binkley, 2001) et d'une utilisation plus complète des 
ressources de l'écosystème (Loreau et al., 2001). En outre, la proportion de feuillus dans les plantations de conifères pourrait accélérer la fermeture du couvert et réduire ainsi la pression sur les ressources du sol en limitant l'abondance des espèces herbacées et d'arbustes bas. Des études ont également démontré l'effet négatif à court terme des traitements sylvicoles (par exemple dégagement de plantation et l'éclaircie précommerciale) sur plusieurs espèces animales (Bujold, 2004). À plus long terme, on craint les effets potentiels sur la biodiversité dus à la raréfaction à l'échelle du paysage des peuplements denses, et ce à différents stades de développement.

Bien que reconnu implicitement par les aménagistes, le caractère mixte des plantations de l'Abitibi n'a pas été documenté rigoureusement. L'utilisation de la télédétection peut s'avérer complémentaire aux inventaires de terrain compte tenu de son faible coût pour de grands territoires (Wulder et al., 2008; Valeria et al., 2012). De plus, de tels outils faciliteraient le suivi de la succession forestière des plantations et permettraient d'établir où et quand il serait justifié d'appliquer des traitements sylvicoles afin de concentrer la croissance sur les espèces visées. En d'autres mots, les méthodes de télédétection sont bien adaptées pour détecter les changements dans l'espace et le temps du couvert des plantations. De nombreuses approches ont déjà été utilisées pour détecter et suivre les changements multi-temporels des surfaces forestières. Le rapport entre bandes spectrales, les indices de végétation (ex. : Indice de Végétation de la Différence Normalisée ou Indice Tasseled), l'analyse en composantes principales et les classifications multidates suivies par l'analyse des changements sont les techniques de télédétection les plus communément employées pour la détection des changements de couverture forestière.

Le but de ce projet était de caractériser les changements de couvert forestier des plantations résineuses sur sol argileux en milieu boréal. Des indices de végétation extraits des images satellitaires Landsat (hiver et été) ont été testés pour leur performance à discriminer la composition du couvert dans les plantations. À plus long terme, ce projet vise à mieux comprendre la succession forestière dans les plantations et évaluer ses impacts sur la possibilité forestière.

\section{Mise en contexte}

\section{Enjeux forestiers}

Au Québec, la remise en production par voie de plantation résineuse a débuté au début des années 1980 et a atteint un sommet en 1990 alors qu'une superficie de plus de 116500 ha a été régénérée artificiellement (Parent, 2009). Le reboisement constitue un complément à la régénération naturelle après coupe, permettant la remise en production des sites peu ou mal régénérés. Les espèces résineuses les plus reboisées au Québec sont l'épinette noire (Picea mariana Mill., Briton), le pin gris (Pinus banksiana Lamb.), le mélèze (Larix laricina (Du Roi) K.Koch), l'épinette blanche (Picea glauca (Moench) Voss) et le pin rouge (Pinus resinosa Aiton). La productivité des plantations dépend de plusieurs facteurs, dont la qualité des plants et de la mise en terre, du choix de la bonne espèce au bon endroit, de la qualité du sol et du drainage, mais surtout de la compétition (Thiffault et al., 2013). Dans la forêt boréale, des espèces de transition s'installent rapidement à la suite d'une coupe forestière notamment les éricacées, les arbustes bas comme le framboisier (Rubus idaeus L.), l'épilobe (Epilobum angustifolium L.), les feuillus de lumière comme le cerisier de Pennsylvanie (Prunus pensylvanica L. f.), le peuplier faux-tremble (Populus tremuloides Michx) et le bouleau à papier (Betula papyrifera Marshall). Ces espèces font concurrence aux arbres plantés en s'appropriant la lumière, les éléments nutritifs et l'eau (Grondin et al., 2003).

Vu l'importance de l'investissement sylvicole, l'évolution du couvert forestier dans les plantations s'avère un élément clé dans la gestion des ressources forestières. Les méthodes les plus courantes utilisées pour déterminer la composition des espèces sont l'inventaire de terrain et l'interprétation photographique, et plus récemment la télédétection. Les mesures sur le terrain sont onéreuses et ne permettent pas de couvrir complètement de grands territoires. Par ailleurs, la télédétection fournit des données spectrales en continu sur des territoires de plusieurs centaines de kilomètres carrés.

\section{Indices de végétation}

Les méthodes de classification de la composition végétale basée sur la télédétection s'avèrent alors efficaces pour obtenir des évaluations du couvert forestier sur de grandes surfaces avec une résolution spatiale à fine échelle. À l'aide de Landsat, les indices de végétation basés sur le rapport de bandes spectrales ont démontré leur efficacité dans la classification de la composition. Ainsi, le rapport PIR/R permettrait une bonne différenciation des types de couvert (Liu et al., 2002) et répondrait aux changements de la biomasse verte, au contenu en chlorophylle et au stress en eau des feuilles (Treitz et Howarth, 1996). Rapide à obtenir, il a cependant un faible pouvoir discriminant quand le recouvrement est inférieur à $50 \%$ et il a une forte dépendance angulaire (Caloz et Collet, 2001). Un autre rapport, MIR(TM5)/MIR(TM7) donnerait une faible différenciation des types de couverts (Liu et al., 2002). Toutefois, lorsqu'il est inversé il présenterait une forte corrélation avec l'humidité du sol (Treitz et Howarth, 1996). L'indice de végétation par différence normalisée (Normalized Difference Vegetation Index - NDVI) est le plus utilisé (Pettorreli, 2013). La valeur du NDVI et de ses dérivés augmente avec la concentration de chlorophylle et la biomasse (Hall et al., 2000). Cet indice permettrait une grande différenciation des résineux en hiver (Liu et al., 2002). L'indice étant indépendant du relief, les effets d'éclairement et de pente sont réduits, par contre il sature en présence d'une épaisse couverture végétale (Caloz et Collet, 2001). Cet indice serait efficace pour détecter et quantifier les changements à grande échelle (Meng et al., 2009). Cependant, il est limité par sa saturation et son insensibilité à certains changements d'indice foliaire (Mcallister et Valeo, 2007). Parmi tous les dérivés, le NDVIc (NDVI corrigé) est reconnu pour sa sensibilité à la biomasse de la forêt (Wulder et Franklin, 2007 ) et il serait plus performant que les autres indices disponibles, car il utilise la bande MIR comme facteur d'échelle (Mcallister et Valeo, 2007). Un autre indice, l'indice Normalized Distance semblerait moins efficace que le NDVI (Mcallister et Valeo, 2007). D'autres indices tenant compte du sol sont également disponibles, notamment l'indice de végétation ajustée pour le sol (Soil Adjusted Végétation Index - SAVI). Cet 
indice permettrait de déterminer la biomasse végétale, cependant il serait limité par la difficulté de calculer la droite des sols très précisément lorsque ceux-ci sont dépourvu de végétation (Caloz et Collet, 2001). Un autre indice, l'indice d'humidité par différence normalisée (Normalized Difference Water Index NDWI) permettrait la distinction entre les feuilles larges et les aiguilles. Cet indice a été utilisé conjointement avec le NDVI, mais il demande plus de validation terrain afin d'évaluer sa capacité à caractériser l'évolution de l'état de la forêt. (Xiao et al., 2002). La transformation Tasseled Cap est un indice efficace qui permet de visualiser la brillance (brightness - B), la verdeur (greenness - G) et l'humidité (wetness - W) de la couverture du sol. Cet indice est sensible à la chlorophylle et serait donc directement relié à la quantité de végétation verte dans un pixel. L'utilisation de cet indice sur plusieurs périodes a donné de bons résultats pour détecter une gamme de changements forestiers (Dymond et al., 2002). De plus, les bandes spectrales dans le proche infrarouge permettraient aussi identifier les espèces dans de jeunes plantations (Olsson 2009).

\section{Méthodologie}

\section{Zone d'étude}

La zone d'étude couvre près de $250 \mathrm{~km}^{2}$ et se situe entre $78^{\circ} 11^{\prime}$ et $79^{\circ} 16^{\prime}$ de longitude Ouest et $49^{\circ} 00^{\prime}$ et $49^{\circ} 32^{\prime}$ de latitude Nord (Figure 1). Le climat de la région est de type continental marqué par de grandes amplitudes de température. Les hivers y sont très froids et les étés relativement chauds (température annuelle moyenne $0.13^{\circ} \mathrm{C}$ ). Les précipitations totalisent annuellement en moyenne $906 \mathrm{~mm}$, dont $24 \%$ tombent sous forme de neige (Gouvernement du Québec, 2015). Le secteur d'étude est localisé dans le biome de la forêt boréale, dans les zones de végétation de la forêt mélangée et de la forêt boréale continue, dans les domaines bioclimatiques de la sapinière à bouleau jaune (Betula alleghaniensis Britton), de la sapinière à bouleau blanc (Betula papyrifera Marshall) et de la pessière à mousse (Veillette et Ducruc, 1983). La végétation de la région est modelée par les feux, les épidémies d'insectes et les chablis, auxquels se sont ajoutées plus récemment les coupes forestières (Bergeron et al., 2014). Le secteur d'étude fait partie du Bouclier canadien et l'assise rocheuse de la région est recouverte de dépôts glaciaires mis en place au moment de la dernière déglaciation (Veillette et Ducruc, 1983). Le dépôt de till (dépôt de surface formé d'un mélange de roches broyées dans une matrice fine et laissées en place lors du passage des glaciers) tapisse les dépressions et est masqué par des dépôts d'argile qui s'étendent en général sous les $300 \mathrm{~m}$ d'altitude (Veillette et Ducruc 1983). Les parcelles étudiées sont localisées sur des dépôts de surface de type glaciolacustre, faciès d'eau profonde (argile). Les pentes sont nulles à faibles (entre $0 \%$ et $8 \%$ ), les classes de drainages selon l'inventaire provincial variant de modéré à imparfait.

Au moment de l'étude, la zone d'étude faisait l'objet d'un contrat d'approvisionnement et d'aménagement forestier alloué à la compagnie Tembec. La superficie totalise 31506 ha soumis à la plantation entre 1980 et 1995 (Tableau 1) dont 18797 ha ont finalement été retenus pour l'analyse. Basée sur les informations disponibles auprès de la compagnie Tembec, la sélection des plantations tenait compte des polygones supérieurs à 2 ha, du type de sol (argileux), du type de plantation (racines nues - PLN, en contenant - PLR, semis - ENS, regarni - PRR ou non-déterminé - $P$ ) et de l'espèce (épinette blanche, épinette noire, mélèze, pin gris, pin rouge) ainsi que du type écologique, du drainage et la pente.

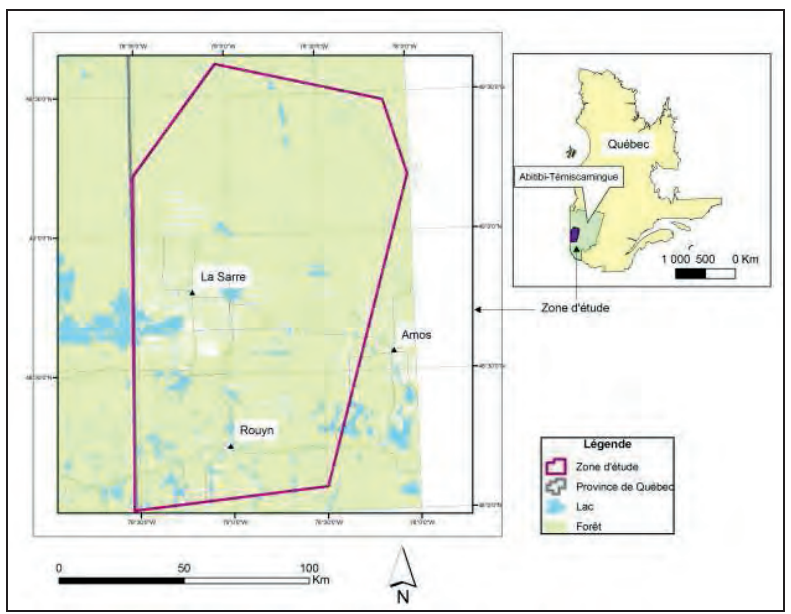

Figure 1 : Localisation de la zone d'étude.

\begin{tabular}{|c|c|c|}
\hline Année & Total (ha) & Groupes (ha) \\
\hline 1980 & 8 & \multirow{6}{*}{$\begin{array}{c}\text { Vieilles } \\
\text { plantations } \\
4507\end{array}$} \\
\hline 1981 & 424 & \\
\hline 1982 & 937 & \\
\hline 1983 & 1137 & \\
\hline 1984 & 1006 & \\
\hline 1985 & 995 & \\
\hline 1986 & 1445 & \multirow{5}{*}{$\begin{array}{c}\text { Plantation } \\
\text { intermédiaires } \\
13592\end{array}$} \\
\hline 1987 & 3331 & \\
\hline 1988 & 3648 & \\
\hline 1989 & 2342 & \\
\hline 1990 & 2826 & \\
\hline 1991 & 4025 & \multirow{5}{*}{$\begin{array}{c}\text { Jeunes } \\
\text { plantations } \\
13407\end{array}$} \\
\hline 1992 & 3797 & \\
\hline 1993 & 3156 & \\
\hline 1994 & 1323 & \\
\hline 1995 & 1106 & \\
\hline Total (ha) & 31506 & 31506 \\
\hline
\end{tabular}

Tableau 1 : Superficie totale (ha) des plantations selon l'année d'établissement.

\section{Images satellites et prétraitements}

Afin de suivre l'évolution des plantations dans le temps, des images satellites Landsat 7 TM et ETM+ avec un couvert nuageux inférieur à $10 \%$ (row 16 et path 28) prises en été (fin juin) et en hiver (fin janvier) 2000, 2005 et 2010 ont été acquises. Les images obtenues ont été ortho rectifiées en utilisant 20 points de contrôle par image suivant la procédure de Wulder et al., (2002) selon le système de projection NAD 1983 UTM zone 17. Une image topographique $1: 20000$ géoréférencée et un fichier MNT ont été utilisés de façon à ce que les images soient superposables entre elles. L'erreur quadratique moyenne (RMS) de géolocalisation se situait entre 10 à $20 \mathrm{~m}$. Une correction de réflectance (Top-of-Atmosphere - TOA) basée sur la théorie de Markham et Barker (1986) a été appliquée aux bandes $1,2,3,4,5$ et 7 . Cette méthode corrige les valeurs de l'image en fonction des variations d'éclairement solaire, de transmission et de rayonnement atmosphériques, en supposant des conditions atmosphériques uniformes sur l'image (Peddle et al., 2003). Finalement, l'image a 
été transformée en valeurs de réflectance et plusieurs indices de végétation ont été calculés pour les années 2000, 2005 et 2010.

De plus, une correction temporelle a été réalisée sur ces images en prenant comme référence les images de 2005. Des zones invariantes dans le temps en termes de réflectance ont été délimitées : forêt mature, milieux urbains, étendue d'eau. Ces zones ont été sélectionnées à l'aide de la fonction "scatterplot" de PCl Geomatica (PCl-Geomatica, 2012) afin d'ajuster les réflectances à l'aide des équations pour 2000-2005 et 2005-2010. Pour les images prises l'hiver et pour la bande TM5, nous avons écarté les étendues d'eau, qui sont alors glacées et couvertes de neige, car cette bande est très sensible aux taux d'humidité de la surface. Les figures 2 et 3 montrent les images satellites de 2005 été et hiver dans un composé coloré de bandes 4-5-3, avec la délimitation du masque de travail et les parcelles étudiées.

Méthodes de classification et de validation de la classification

A partir des images satellites une classification automatisée par seuillage basée sur nos observations a été réalisée pour chaque période pour les indices de végétation et variables suivantes: Tasseled Cap Brightness été, différence NDVIc été moins hiver, Tasseled Cap Greenness été et NDVIc hiver. Ces indices sont considérés les plus discriminants selon Hall et al., (2000). La classification a été réalisée au niveau du pixel à l'aide des modules $\mathrm{PCl}$ Modeller et PCI EASI du logiciel PCI Geomatica.

Les seuils des indices ont été établis en s'aidant des inventaires écoforestiers décennaux du ministère des Ressources naturelles du Québec disponibles. Les types de couvert ont été identifiés pour chaque période sur des mosaïques d'ortho-photographies panchromatiques et couleur au 1:20000 (voir exemple pour 2005, Figure 4). Les types de couvert ont été établis en suivant le protocole de la direction des inventaires forestiers au Québec, en fonction du pourcentage de surface terrière du peuplement ( $F$ lorsque feuillus > $75 \%$, R lorsque $>75 \%$ résineux et le complément $M$ pour les peuplements mixtes). Les valeurs des indices de végétation pour chaque période ont été alors comparées avec le type de couvert afin de différencier les classes obtenues (Figure 5).

Nous avons vérifié chaque classification en utilisant 56 points par période d'analyse à partir des ortho photographies panchromatiques et couleur au $1: 20000$ disponibles pour chaque période auprès de la direction d'inventaire forestier au Québec. De plus, afin de vérifier la réalité terrain de résultats de 2010, des points d'observation terrain ont été réalisés en 2011 dans 54 peuplements choisis aléatoirement. Les points étaient localisés au centre du peuplement afin d'éviter les effets bordure et de la route. Sur le terrain, la hauteur et l'âge de la classe dominante ou codominante représentative de la plantation, la classe de diamètre, la classe de densité, la surface terrière estimée avec un prisme forestier $(F A B=2)$ et le pourcentage de feuillus (dans un rayon de 10 mètres) ont été recueillis. D'autres observations telles que la présence de mousses et de sphaignes sur le sol, les indices des traitements sylvicoles (dégagement éclaircies pré-commerciale) et l'aspect général de la plantation ont été notés.

Une matrice de confusion a été générée en superposant les points d'observation terrain à l'image classifiée en 2010 en utilisant ArcView (ESRI, 2010). Nous avons utilisé la statistique Kappa qui quantifie la précision globale par rapport au hasard dans un tableau de contingence (Czaplewski, 2000).

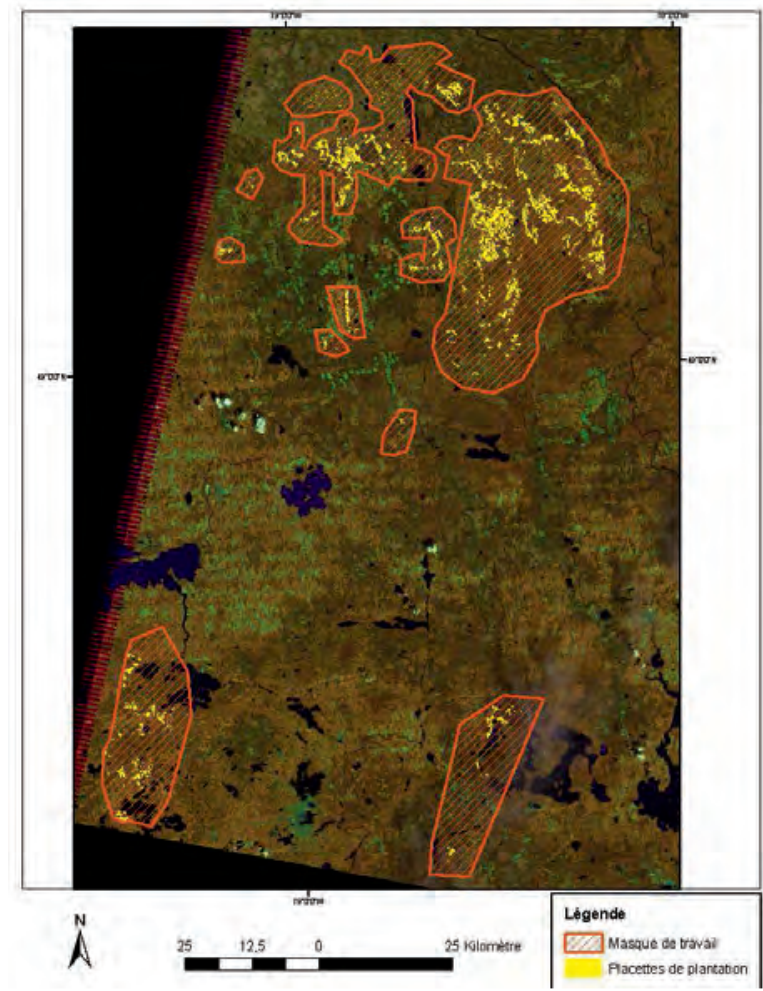

Figure 2: Image Landsat pour la période été 2005 et localisation de masque de travail (rouge) et secteurs de plantation (jaune).

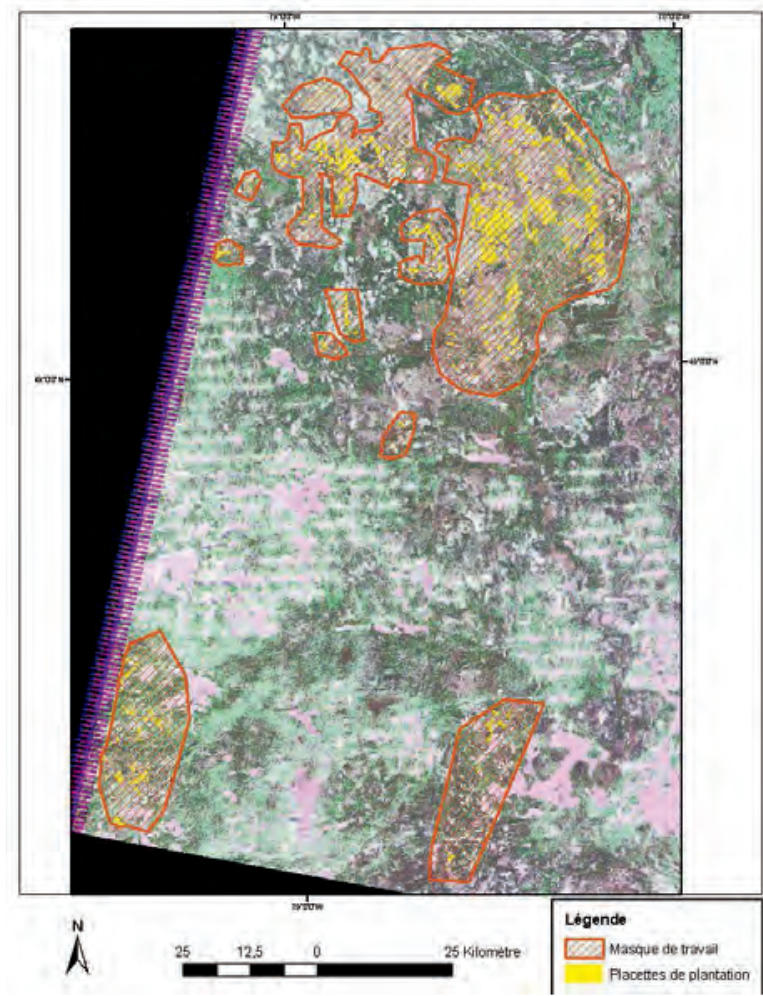

Figure 3 : Image Landsat période hiver 2005 avec localisation du masque de travail (rouge) et des secteurs de plantation (jaune). 

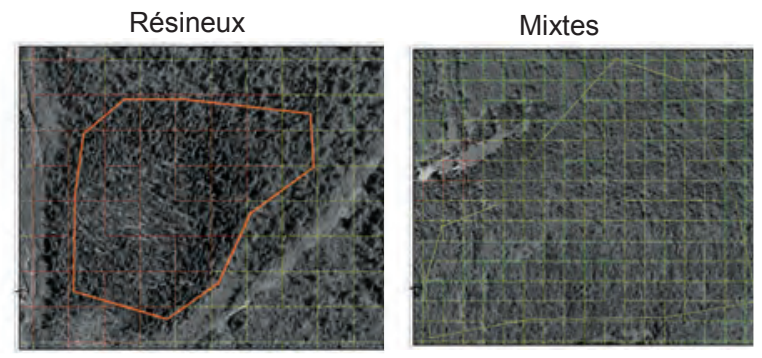

Feuillus

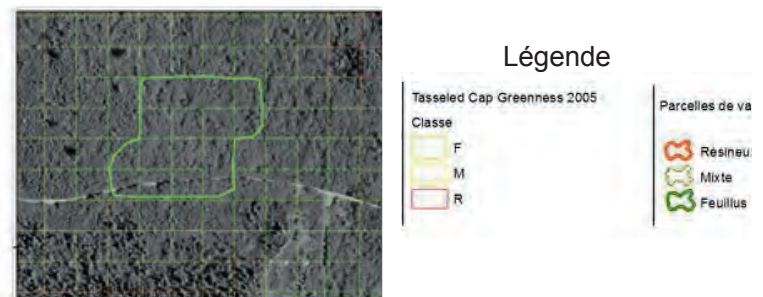

Figure 4 : Secteurs représentés par une grille régulière colorée (rouge, jaune et vert respectivement $R, M$ et $F$ ) selon I'Indice Tasseled Cap Greenness en 2005 superposé aux secteurs de l'ortho photo 2007 pour validation (rouge $=R$ résineux, jaune $=M$ mixtes et vert $=F$ feuillus).

\section{Méthode d'analyse de l'évolution forestière}

Finalement, un modèle généralisé linéaire mixte avec approximation de Laplace a été utilisé pour déterminer la probabilité de changement de couvert en fonction de l'âge de plantation (maximum 25 ans). Cette probabilité de changement de couvert en fonction de l'âge a été exprimée en fonction :

1) du type initial de couvert basé sur la classification ( $M$, $R, F)$,

2) de l'âge de la plantation au moment de l'évaluation par télédétection du type de couvert (date d'évaluation 2000, 2005 et 2010),

3) du type de plantation (PLN, PLR, ENS, PRR ou P),

4 ) de l'interaction type de couvert initial détecté par la classification,

5) d'un effet aléatoire associé à l'identifiant du polygone de la plantation.

La probabilité de changement (Probabilité = Type initial + âge + type de plantation + type initial : âge + (1/ID plantation) a été évaluée avec le logiciel $R$ ( $R$ Development Core Team 2005).

\section{Résultats}

\section{Cartographie par télédétection}

L'indice Tasseled Cap Greenness a été retenu pour présenter l'évolution du type de couvert des plantations compte tenu de sa meilleure discrimination entre les types de couvert (Figure 5). De plus, l'indice Tasseled Cap Greenness a été de loin supérieur (kappa $=50 \%$ Tableau 2) comparés au Tasseled Cap Brightness et NDVIc différence été moins hiver (kappa $47 \%$ et $36 \%$, respectivement, tableau non présenté ici) pour la validation terrain 2010. La même tendance a été observée avec les validations réalisées à partir des ortho photographies panchromatiques et couleur au 1:20000 pour chaque période. Ainsi, les performances de la classification selon l'indice Tasseled Cap Greenness étaient de $48 \%$ et $37 \%$ (Kappa), respectivement pour 2000 et 2005 (supérieurs aux autres classifications) (Figure 6). Un exemple de la variabilité spatiale de l'indice pour 2000, 2005 et 2010 de chaque type de couvert résineux $(R)$, mixte $(M)$ et feuillu $(F)$ est présenté sur une partie de la zone d'étude (Figure 7).

\section{Tasseled Cap Brightness}

\section{NDVIc différence été} moins hiver

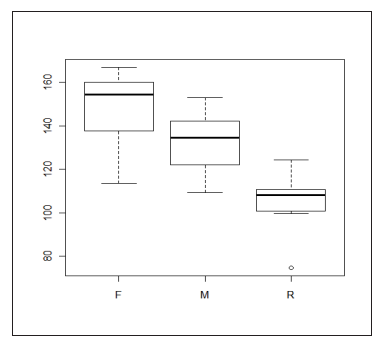

Tasseled Cap Greenness été

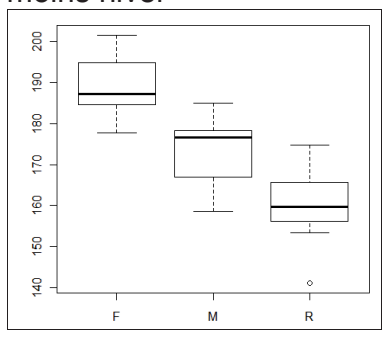

NDVIc hiver
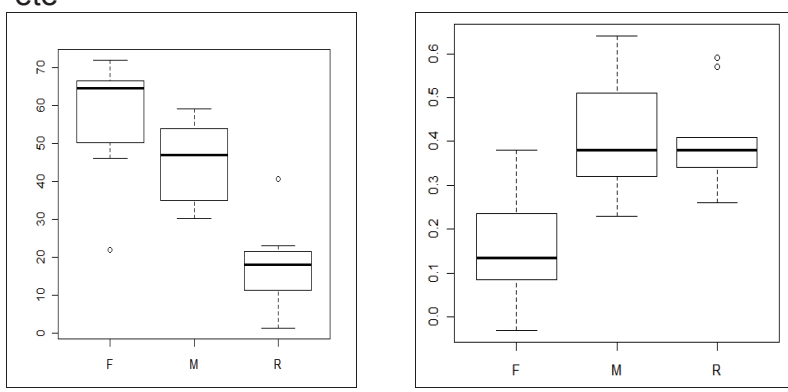

Figure 5: Graphique boite à moustache de quatre classification des indices, pour l'image 2005 en fonction du type de couvert ( $F$ feuillu, $M$ mixte et $R$ résineux).

Le portrait d'une superficie de 18797 ha élaboré à partir de l'indice Tasseled Cap Greenness montre la transition du type de couvert en pourcentage et en hectare de chaque pixel entre 2000 et 2010 soit entre 5 et 30 ans après l'établissement des plantations (Tableau 3). Selon cet indice de végétation, les peuplements mixtes dominent avec des superficies de 9 322, 11261 et 9664 ha en 2000, 2005 et 2010, respectivement. Ils sont suivis par les peuplements résineux couvrant 4736,3178 et 7127 ha pour les années 2000, 2005 et 2010. Finalement, les peuplements feuillus représentent 4739,4358 et 2006 ha pour ces mêmes années.

\begin{tabular}{|c|r|r|r|r|r|r|}
\hline $\begin{array}{c}\text { Points/ } \\
\text { Validation }\end{array}$ & \multicolumn{2}{|c|}{ Classification } & \multirow{2}{*}{ Total } & $\begin{array}{c}\text { Usager } \\
(\%)\end{array}$ & $\begin{array}{c}\text { Commission } \\
(\%)\end{array}$ \\
\hline $\mathrm{F}$ & 13 & 3 & 2 & 18 & 72.2 & 27.7 \\
\hline $\mathrm{M}$ & 3 & 10 & 5 & 18 & 55.5 & 44.4 \\
\hline $\mathrm{R}$ & & 5 & 13 & 18 & 72.2 & 27.7 \\
\hline Total & 16 & 18 & 20 & 54 & & \\
\hline $\begin{array}{c}\text { Producer } \\
(\%)\end{array}$ & 81.2 & 55.5 & 65.0 & 66.6 & & \\
\hline $\begin{array}{c}\text { Omission } \\
(\%)\end{array}$ & 18.7 & 44.4 & 35.0 & & & \\
\hline Kappa & $50.0 \%$ & & & & & \\
\hline
\end{tabular}

Tableau 2 : Matrice de confusion de l'Indice Tasseled Cap Greenness en 2010.

En général, le couvert mixte domine les plantations, toutefois la proportion de couvert résineux croît avec le temps au détriment de couvert feuillu et mixte (Tableau 3 ). L'évolution d'un couvert résineux vers un couvert feuillu ne dépasse pas $4.2 \%$ des superficies pour toutes les périodes. De manière équivalente, les superficies du couvert feuillu qui évoluent vers le couvert résineux demeurent très faibles $(<7.25 \%)$. De 
plus, les couverts résineux identifiés avec l'image 2000 maintiennent en général leur composition, à l'exception de la période 2000 - 2005 pendant laquelle le couvert résineux évolue majoritairement $(56.3 \%)$ vers le couvert mixte.

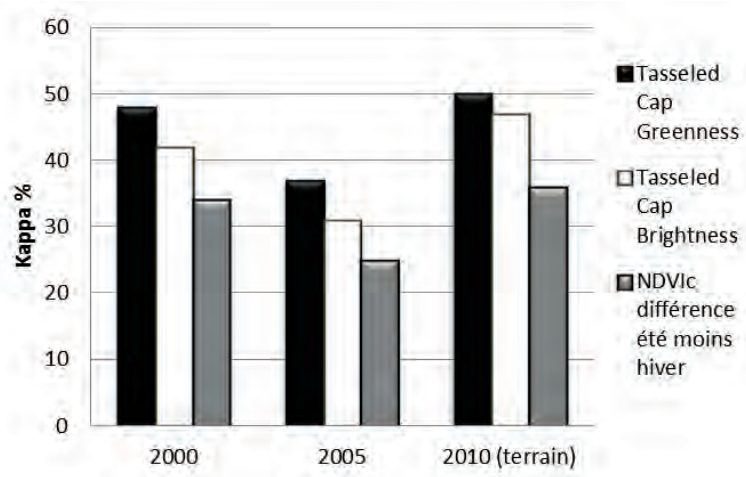

Figure 6: Valeur Kappa de classification par période pour les trois indices les plus performants.

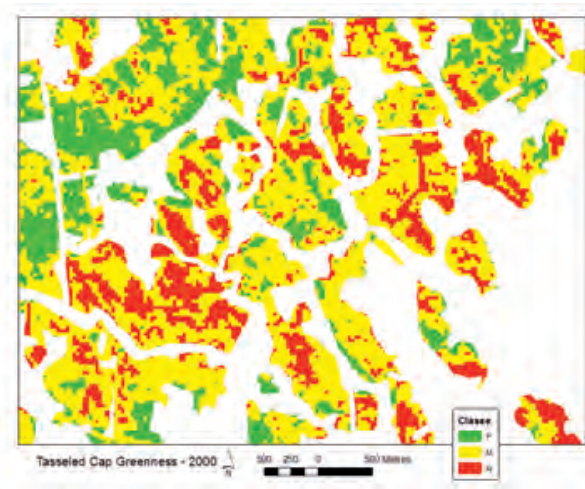

2000
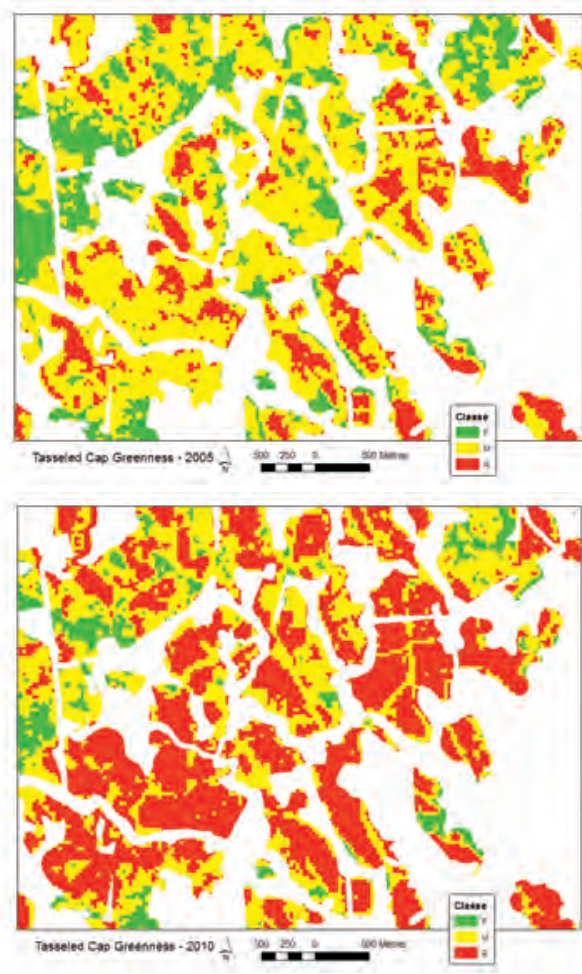

Figure 7: Classification de l'indice Tasseled Cap Greenness de 2000, 2005 et 2010 pour une portion de la région d'étude les couleurs rouge, jaune et vert correspondent respectivement à résineux, mixte et feuillu.

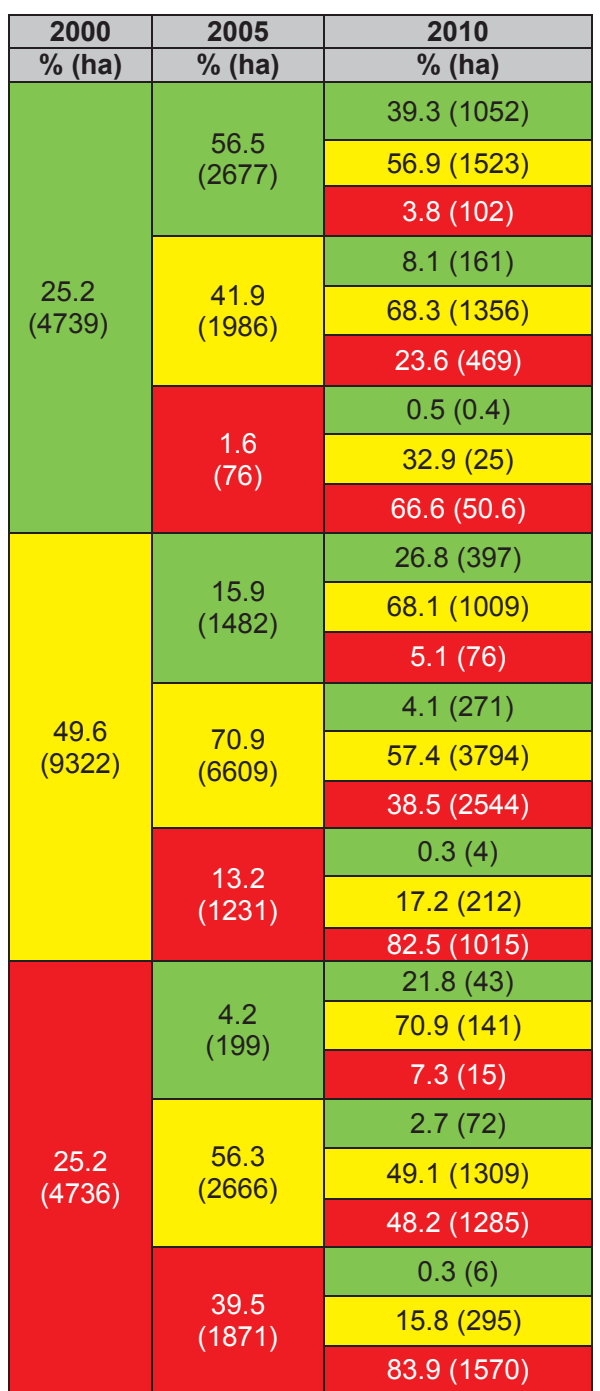

Tableau 3: Matrice de transition du type de couvert feuillu (vert), mixte (jaune) et résineux (rouge) en proportion et en hectare pour les années d'évaluation 2000, 2005 et 2010. Résultats basés sur l'indice Tasseled Cap Greenness.

On observe sur l'ensemble de la période d'analyse que la superficie de couvert résineux a augmenté en 2010 (7 126.6 ha) comparativement aux années 2000 et 2005 , possiblement en raison du meilleur succès des jeunes plantations. En effet, environ 20 ans après leur établissement, la proportion des peuplements résineux est supérieure pour les plantations les plus récentes comparativement aux vielles plantations ( $34.1 \%$ pour la période 1990-1995 contre $7.8 \%$ pour la période 19801985) (Figure 8). Ce portrait pourrait refléter une évolution vers des règles plus strictes encadrant les plantations, expliquée par le succès et dominance des résineux. Nonobstant cette tendance, la proportion de couverts mixtes demeure importante $(53.3 \%)$ environ 20 ans après l'établissement, ce qui pourrait devenir préoccupant pour les aménagistes du territoire, d'autant plus que la proportion de couvert feuillu demeure importante dans les plus vielles plantations (pour 1986$199019.5 \%$ et pour 1980-1985 38 \%) (Figure 8).

Facteurs liés au changement de type de couvert forestier 
Les résultats du modèle généralisé linéaire mixte montrent que l'ensemble des variables analysées sont significatives voire hautement significatives (Tableau 4). La matrice de corrélations met en lumière l'interaction entre le couvert et l'âge de la plantation (Tableau 5).

\begin{tabular}{|c|c|c|c|c|}
\hline Groupes & Nom & Variance & \multicolumn{2}{|c|}{ Std.Dev. } \\
\hline ID_planta & (Intercept) & \begin{tabular}{l|l} 
& 0.88547 \\
\end{tabular} & \multicolumn{2}{|c|}{0.94099} \\
\hline \multicolumn{5}{|c|}{ Nombre d'observations: 25972 , groupes: ID_plantation, 5580} \\
\hline Effets fixes & Estimation & Std. Error & Valeur Z & $\operatorname{Pr}(>|z|)$ \\
\hline (Intercept) & -10.943507 & 0.070801 & -154.57 & $<2 \mathrm{e}-16^{* \star \star}$ \\
\hline Type.initM & 3.403338 & 0.034911 & 97.49 & $<2 \mathrm{e}-16^{* * *}$ \\
\hline Type.initR & 11.159743 & 0.041710 & 267.55 & $<2 \mathrm{e}-16^{* * *}$ \\
\hline Age & 0.458828 & 0.001482 & 309.60 & $<2 \mathrm{e}-16^{* \star *}$ \\
\hline TYPE_P & 1.272520 & 0.063927 & 19.91 & $<2 \mathrm{e}-16^{* * *}$ \\
\hline TYPE_PLN & 1.118263 & 0.346264 & 3.23 & $0.00124^{* *}$ \\
\hline TYPE_PLR & 1.708249 & 0.063853 & 26.75 & $<2 e-16^{* * *}$ \\
\hline TYPE PRR & 1.748976 & 0.088379 & 19.79 & $<2 \mathrm{e}-16^{* * *}$ \\
\hline Type.initM:Age & -0.220196 & 0.001559 & -141.26 & $<2 \mathrm{e}-16^{* * *}$ \\
\hline Type.initR:Age & -0.522572 & 0.001863 & -280.44 & $<2 \mathrm{e}-16^{* * *}$ \\
\hline
\end{tabular}

Tableau 4 : Résultats du modèle à effets fixes pour chaque variable où Type.init $M$ (couvert de départ mixte), Type.init $R$ (couvert de départ résineux), Age (âge de la plantation au moment de la détection), type de plantation (Type PLN racines nues, Type PLR en contenant, Type PRR regarni et TypeP non-déterminé) et interactions (Type.init M:Age et Type.init R:Age).

Ainsi, la probabilité de changement de couvert d'une plantation est plus importante si le couvert initial est dominé par les feuillus (68\%) ou un couvert résineux (52\%) (Figure 9). Toutefois, la probabilité de changer évolue selon l'âge de la plantation (Figure 10). Ainsi, le couvert classé feuillus ou mélangés présente une probabilité de changement qui s'accroît avec l'âge de la plantation. Le taux de changement de cette probabilité de couvert est maximal entre 20 et 25 ans après établissement pour le couvert feuillu avec un plafond aux alentours de 30 ans, tandis que pour le couvert mixte cette probabilité de changement augmente constamment entre 15 et 35 ans passant de $10 \%$ à $83 \%$ (Figure 10). À l'opposé, le couvert résineux présente une probabilité de changement de $65 \%$ qui diminue jusqu'à $30 \%$ à 35 ans (Figure 10). Ces données suggèrent qu'entre 20 et 25 ans après la plantation, l'éducation des peuplements est plus critique, et que la présence des espèces de lumière est très importante même après 30 ans dans le cas des plantations résineuses.

\begin{tabular}{|l|r|r|r|r|r|r|r|r|}
\hline & $\begin{array}{c}\text { Inter- } \\
\text { cept }\end{array}$ & $\begin{array}{l}\text { Type. } \\
\text { init M }\end{array}$ & $\begin{array}{l}\text { Type. } \\
\text { init R }\end{array}$ & Age & $\begin{array}{c}\text { TYPE } \\
\text { P }\end{array}$ & $\begin{array}{r}\text { TYPE } \\
\text { PLN }\end{array}$ & $\begin{array}{r}\text { TYPE } \\
\text { PLR }\end{array}$ & $\begin{array}{r}\text { TYPE } \\
\text { PRR }\end{array}$ \\
\hline Type.init M & -0.38 & & & & & & & \\
\hline Type.init R & -0.36 & 0.67 & & & & & & \\
\hline Age & -0.51 & 0.79 & 0.723 & & & & & \\
\hline TYPE_P & -0.84 & 0.01 & 0.027 & 0.05 & & & & \\
\hline TYPE_PLN & -0.16 & 0.01 & 0.008 & 0.01 & 0.17 & & & \\
\hline TYPE_PLR & -0.85 & 0.01 & 0.028 & 0.07 & 0.91 & 0.17 & & \\
\hline TYPE_PRR & -0.62 & -0.001 & 0.004 & 0.06 & 0.66 & 0.12 & 0.66 & \\
\hline Type.init M:Age & 0.38 & -0.98 & -0.668 & -0.82 & -0.01 & -0.004 & -.012 & -0.004 \\
\hline Type.init R:Age & 0.37 & -0.66 & -0.981 & -0.75 & -0.03 & -0.008 & -0.03 & -0.011 \\
\hline
\end{tabular}

Tableau 5 : Matrice de corrélation entre les variables du model (effets fixes) où Type.init $M$ (couvert de départ mixte), Type.init $R$ (couvert de départ résineux), Age (âge de la plantation au moment de la détection), type de plantation (Type PLN racines nues, Type PLR en contenant, Type PRR regarni et TypeP non-déterminé) et interactions (Type.init M:Age et Type.init R:Age).

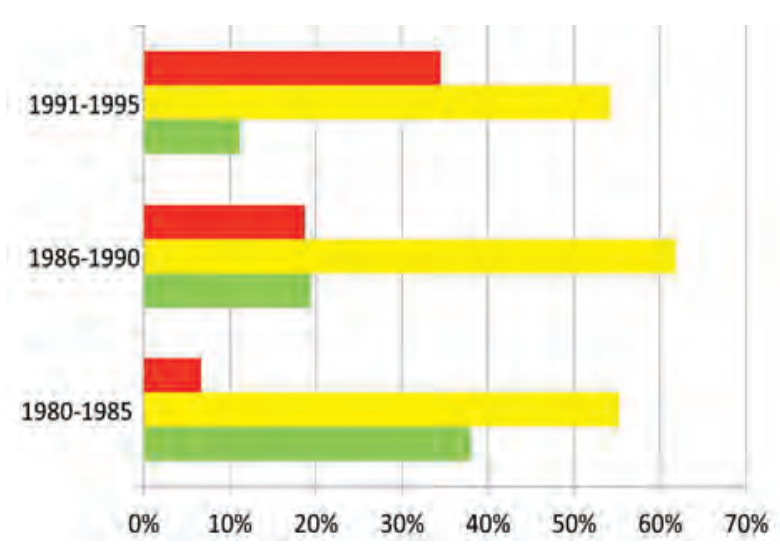

Figure 8: Proportion du type de couvert (résineux rouge, mixte - jaune et feuillu - vert) selon l'Indice Tasseled Cap Greenness environ 20 ans après l'établissement pour des plantations

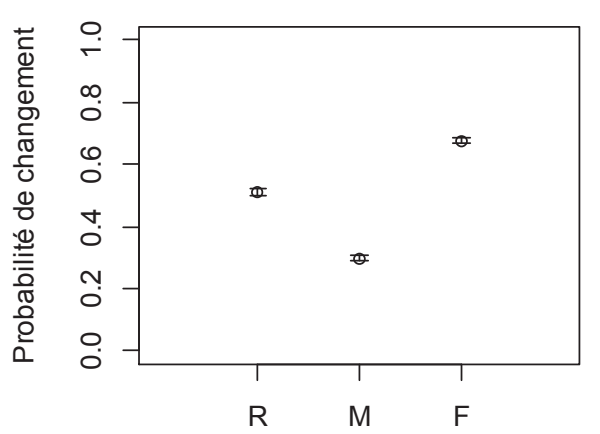

Type de peuplement initial

Figure 9 : Probabilité de changement (absolu) en fonction du type de peuplement initial ( $R=$ résineux, $M=$ mixte et $F=$ feuillu).

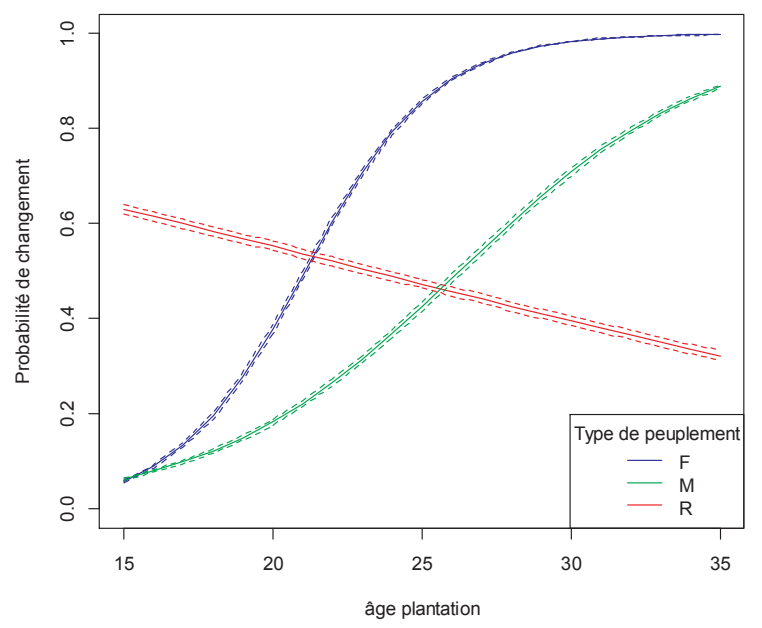

Figure 10 : Évolution de la probabilité de changement (absolu) par type de peuplement ( $R=$ résineux, $M=$ mixte et $F=$ feuillu) en fonction de l'âge de la plantation. 


\section{Discussion}

Cartographie par télédétection

La performance d'indices de végétation, tels NDVI, NDVIc, NDWI et Tasseled Cap a été signalée par plusieurs auteurs (Peddle et al., 2001; Mcallister et Valeo, 2007; Wulder et Franklin, 2007; Olsson, 2010; Pettorelli, 2013). Nous avons aussi utilisé l'indice NDVIc calculé à partir d'images satellites en été et hiver afin de maximiser le contraste (feuillu et résineux). Toutefois, l'indice Tasseled Cap Greenness a été supérieur $(\mathrm{kappa}=50 \%$ ) aux autres classifications. Ceci peut être expliqué par sa capacité à mieux détecter les changements de couvert et à différencier la structure du couvert végétal (Dymond et al., 2002; Healey et al., 2005). En effet, les différences observées dans les propriétés spectrales des plantations pourraient être dues à des différences dans la chimie ou la structure des feuilles, ou dans le contenu en eau parmi les espèces qui caractérisent chacune des classes de succession de la forêt (Vieira et al., 2003). La performance de la classification 2005 était inférieure ce qui peut expliquer la confusion entre les classes $M$, $F$ et $R$.

Nous avions aussi à notre disposition une classification hybride avec la méthode ECM (Enhancement Classification Method) (Valeria et al., 2012), cependant cette classification présentait un niveau d'erreur plus élevé. Toutefois, dans d'autres études, cette classification ECM a obtenu plus de $90 \%$ de réussite en utilisant des images multi-temporelles (Liu et al. 2002 ) et plus de $84 \%$ de réussite pour déterminer les surfaces forêt et non forêt (Valeria et al., 2008). Elle a eu aussi un très haut taux de correspondance pour 25 classes de structure forestière (Peddle et al., 2004). Les classifications supervisées, de leur côté, offrent une bonne fiabilité, cependant nous n'avions pas un nombre suffisant de donnés pour les mettre en application. D'autres méthodes de classification "fuzzy » (Maselli et al., 1995) ou basées sur les objets intégrant des propriétés radiométriques semblent très prometteuses (Qin et al., 2013) et devraient aussi être expérimentées. De plus, de nouveaux capteurs (Sentinel 2) pourraient améliorer la performance de la classification, grâce à un nombre plus élevé des bandes spectrales, une meilleure précision, une résolution et une périodicité accrues permettant ainsi une intégration des outils de télédétection dans le suivi de plantations.

\section{Evolution forestière}

Les plantations forestières constituent un élément fondamental de toute stratégie d'approvisionnement forestier afin de répondre aux attentes de la société en matière d'aménagement forestier durable (Thiffault et al., 2003). Nonobstant l'intérêt de maintenir et accroître la productivité des peuplements purs résineux, les nouvelles stratégies d'aménagement inspirées des patrons de perturbation naturelle nous amènent à nous questionner sur la valeur écologique de ces plantations. En effet, les pressions du public face à l'artificialisation de la forêt sont importantes et forcent les aménagistes à moduler la taille, la composition et l'agencement des plantations dans le paysage (Barrette et al., 2014). Ainsi, un système de suivi basé sur l'analyse d'images satellites permettrait non seulement d'identifier où et quand intervenir par un traitement sylvicole, mais aussi d'identifier les plantations qui ne requièrent pas d'intervention de manière à respecter leur dynamique naturelle (Barrette et al., 2014). II faciliterait aussi la gestion de la composition à l'échelle du paysage.

\section{Conclusion}

L'utilisation de la télédétection par images satellite afin de suivre l'évolution du couvert de plantations de résineux, évaluée ici sur une période d'analyse de 15 ans semble prometteuse. Après une revue de littérature qui a permis de sélectionner certains indices de végétation, nous avons déterminé les mieux adaptés à notre zone d'étude. Les indices: Tasseled Cap brightness et Greenness en été, ainsi que la différence NDVIc été moins hiver se sont avérés les plus intéressants pour discriminer le type de couvert. Toutefois, l'indice Tasseled Cap Greenness s'est avéré le plus performant avec un indice de Kappa de $50 \%$. Un portrait des plantations établies entre 1980 à 1995 a été effectué avec l'indice Tasseled Cap Greenness pour les années 2000, 2005 et 2010. Les résultats montrent que le couvert de plus de $50 \%$ des plantations étant classifié mixte et $25 \%$ était classifié feuillu en 2000 alors qu'en 2010, $51 \%$ des plantations étaient mixtes et $11 \%$ étaient feuillues. Toutefois, les résultats montrent que les changements de composition varient selon l'âge de la plantation surtout pour les couverts feuillu et mixte. La probabilité de changement d'un couvert initial feuillu vers un autre type de couvert est près de $68 \%$. Les variables types de plantation, âge de la plantation et type couvert initial ainsi que leurs interactions sont toutes significatives pour déterminer le changement de type de couvert.

Les résultats offrent un portrait d'ensemble de l'évolution de plus de 18797 ha de plantations. Compte tenu des résultats, les outils de télédétection s'avèrent efficaces pour le suivi d'un territoire de cette dimension. Un tel suivi permettrait de mieux cibler des traitements sylvicoles en fonction d'objectifs d'aménagement spécifiques ou d'ajuster le calcul de la possibilité forestière à la composition réelle des plantations.

\section{Remerciements}

Cette étude a été financée par le programme de mise en valeur des Ressources du milieu forestier - volet I du ministère de Ressources naturelles et de la faune du Québec. Les auteurs remercient la collaboration de la compagnie forestière Tembec pour leur contribution à la réalisation de ce projet. Les auteurs remercient également les commentaires d'évaluateurs anonymes ayant permis d'améliorer la qualité de cet article.

\section{Références}

Barrette M., M. Leblanc, N. Thiffault, A. Paquette, L. Lavoie, L. Bélanger, F. Bujold, L. Côté, J. Lamoureux, R. Schneider, J.-P. Tremblay, S. Côté, Y. Boucher, M.-Ė. Deshaies 2014. Issues and solutions for intensive plantation silviculture in a context of ecosystem management. Forestry Chronicle 90(6):748-762.

Bergeron Y., H. Y.H. Chen, N. C. Kenkel, A. L. Leduc, S. et E. Macdonald 2014. Boreal mixedwood stand dynamics: ecological processes underlying multiple pathways. The Forestry Chronicle 90(2): 202-213. 
Bujold, F., A. Cimon, P. Blanchette, M. Crête Et J. Legris, 2004. Effets de l'éclaircie précommerciale sur la diversité biologique: document de support justifiant un objectif de protection et de mise en valeur des ressources du milieu forestier, 16 p. (non publié).

Caloz R. et Collet C. 2001. Précis de télédétection, Vol. III : Traitements numériques d'images de télédétection, Presses de I'Université du Québec / AUPELF, $386 \mathrm{p}$.

Czaplewski, R. J. 2000. Accuracy assessments and areal estimates using two phase Stratified Random Sampling cluster plots and the Multivariate Composite Estimate. In H. T. Mowrer, \& R. G. Congalton (Eds.), Quantifying spatial uncertainty in natural resources (pp. 79 - 100). Chelsea, MI: Ann Arbor Press.

Dymond, C. C., Mladenoff, D. J., et Radeloff, V. C. 2002. Phenological differences in Tasseled Cap indices improve deciduous forest classification. Remote Sensing of Environment, 80(3), 460-472.

ESRI 2010. ArcGIS [GIS software]. Version 10.0. Redlands, CA: Environmental Systems Research Institute, Inc., Redlands, California.

Gouvernement du Québec 2015. Institut de la statistique du Québec. En ligne http://www.stat.gouv.qc.ca/statistiques/

quebec statistique/ter ter/ter ter $6 . \mathrm{htm}$ consulté le 30/01/15

Grondin, P., J.-P. Saucier, J. Blouin, J. Gosselin et A. Robitaille, 2003. Information écologique et planification forestière au Québec, Canada. Note de recherche forestière $n^{\circ}$ 118. Ministère des Ressources naturelles, de la Faune et des Parcs, Direction de la recherche forestière. 10 p.

Hall, R. J., Peddle, D. R., et Klita, D. L. 2000. Mapping conifer understory within boreal mixedwoods from Landsat TM satellite imagery and forest inventory information. Forestry Chronicle, 76(6), 887-902.

Healey, S. P., Cohen, W. B., Zhiqiang, Y., et Krankina, O. N. 2005. Comparison of Tasseled Cap-based Landsat data structures for use in forest disturbance detection. Remote Sensing of Environment, 97(3), 301-310.

Hollingsworth, T.N., Walker, M.D., Chapin III, F.S., et Parsons, A.L. 2006. Scale-dependent environmental controls over species composition in Alaskan black spruce communities. Canadian Journal of Forest Research, 36(7), 1781-1796.

Jobidon, R. 2000. Density-dependent effects of northern hardwood competition on selected environmental resources and young white spruce (Picea glauca) plantation growth, mineral nutrition, and stand structural development - a 5-year study plantation growth, mineral nutrition, and stand structural development - a 5-year study. Forest Ecology and Management, 130, 77-97.

Légaré, S., Bergeron, Y., et Paré, D. 2005. Effect of aspen (Populus tremuloides) as a companion species on the growth of black spruce (Picea mariana) in the southwestern boreal forest of Quebec. Forest Ecology and Management, 208, 211-222.

Liu, Q. J., Takamura, T., Takeuchi, N., et Shao, G. 2002. Mapping of boreal vegetation of a temperate mountain in China by multitemporal Landsat TM imagery. International Journal of Remote Sensing, 23(17), 21p.
Loreau, M., S. Naeem, P. Inchausti, J. Bengtsson, J. P. Grime, A. Hector, D. U. Hooper, M. A. Huston, D. Raffaelli, B. Schmid, D. Tilman et D. A. Wardle 2001. Biodiversity and Ecosystem Functioning: Current Knowledge and Future Challenges. Science 26, 294(5543), 804-808.

Man, R., et Lieffers, V. J. 1999. Are mixtures of aspen and white spruce more productive than single species stands? Forestry Chronicle 75: 505-513.

Markham, B., et Barker, J. (1986). Landsat MSS and TM post calibration dynamic ranges, exoatmospheric reflectances and at satellite temperature. EOSAT Landsat Technical Notes, 1, 3-7.

Mcallister, D. M., et Valeo, C. 2007. A robust new method for the remote estimation of LAl in montane and boreal forests. International Journal of Remote Sensing, 28(8), 15p.

Maselli, F., Conese, C., De Filippis, T. et S. Norcini 1995. Estimation of forest parameters through fuzzy classification of TM data. Geoscience and Remote Sensing, IEEE Transactions 33(1): 77 - 84.

Meng, Q., Cieszewski, C., et Madden, M. 2009. Large area forest inventory using Landsat ETM+: A geostatistical approach. ISPRS Journal of Photogrammetry and Remote Sensing, 64(1), 27-36.

Olsson H. 2009. A method for using Landsat time series for monitoring young plantation in boreal forest. International journal of remote sensing 30:19, 51175131.

Olsson H., Persson A., Björk L., Rosengren M. 2010. Extended Abstract publicerat i Miranda, Suarez, Cresente (Ed.) Operational tools in forestry using remote sensing techniques - Proceedings från ForestSat 2010 134-136.

Paré, D., et Y. Bergeron 1995. Above ground biomass accumulation along a 230 year chronosequence in the southern portion of the Canadian boreal forest. Journal of Ecology., 83, 1001-1008.

Parent, B. 2009. Ressources et industries forestières. Portrait statistiques édition 2009. Gouvernement du Québec. Ministère des Ressources naturelles et de la Faune. URL http://www.mrn.gouv.qc.ca/publications/forets/connais sances/statistiques/resume2009.pdf.

PCl-Geomatica 2012. PCl-Geomatica OrthoEngine User Guide 168 p.

Peddle, D. R., Johnson, R. L., Cihlar, J., et Latifovic, R. 2004. Large area forest classification and biophysical parameter estimation using the 5-Scale canopy reflectance model in Multiple-Forward-Mode. Remote Sensing of Environment, 89(2), 252-263.

Peddle, D., Teillet, P., et Wulder, M. 2003. Radiometric image processing. In M. Wulder, et S. Franklin (Eds.), Remote sensing of forest environments: Concepts and case studies (pp. 181-208). Boston: Kluwer Academic Publishers

Peddle, R., D., Brunke, P., S., Hall, et G., F. 2001. A comparison of spectral mixture analysis and ten vegetation indices for estimating boreal Forest biophysical information from airborne data: Remote sensing and spatial data integration: Measuring, monitoring, and modelling (Vol. 27). Canadian Aeronautics and Space Institute. Ottawa, ON, CANADA. 
Pettorelli N. 2013. The Normalized Difference Vegetation Index. Oxford: Oxford University press. $208 \mathrm{pp}$.

Qin, Y., Z. Niu, F. Chen, B. Li, and Y. Ban. 2013. Object-based land cover change detection for crosssensor images. International Journal of Remote Sensing 34:6723-6737.

Price, D.T., Alfaro, R.I., Brown, K.J., Flannigan, M.D., Fleming, R.A., Hogg, E.H., Girardin, M.P., Lakusta, T., Johnston, M., McKenney, D.W., Pedlar, J.H., Stratton, T., Sturrock, R.N., Thompson, I.D., Trofymow, J.A., et Venier, L.A. 2013. Anticipating the consequences of climate change for Canada's boreal forest ecosystems1. Environmental Reviews, 21, 322-365. dx.doi.org/10.1139/er-2013-0042.

R Development Core Team (2005). R: A language and environment for statistical computing. R Foundation for Statistical Computing, Vienna, Austria. ISBN 3900051-07-0, URL: http://www.R-project.org.

Rothe, A., et Binkley, D. 2001. Nutritional interactions in mixed species forests: a synthesis. Canadian Journal Forest Research 31(11), 1855-1870.

Thiffault N., V. Roy, G. Prégent, G. Cyr, R. Jobidon et J. Ménétrier 2003. La sylviculture des plantations résineuses au Québec. Le Naturaliste Canadien, Vol. 127 No 1 :63-80. Hiver 2003.

Thiffault, N., V. Roy, J. Ménétrier, G. Prégent et A. Rainville 2013. La plantation, dans Larouche, C., et al., Le guide sylvicole du Québec, Tome 2, Les concepts et l'application de la sylviculture, Québec, Les Publications du Québec, p. 197-225

Treitz, P. et P. Howarth 1996. Remote sensing for forest ecosystem characterization: a review. Nat. Resource Canada, Canadian Forest Service, Sault Ste. Marie, NODA/NFP Tech. Rep. TR-12, 51p.
Valeria O., A. Laamrani et A. Beaudoin, 2012. Monitoring the state of a large boreal forest region in eastern Canada through the use of multitemporal classified satellite imagery. Canadian Journal of Remote Sensing 38(1):91-108.

Valeria O., A. Laamrani, B. Laib, A. Beaudoin, S. Côté and G. Simard. 2008. Rapport de l'état de la forêt en Abitibi-Témiscamingue et au Nord-du-Québec par télédétection. Université du Québec en Abitibi Témiscamingue. $67 \mathrm{p}$.

Veillette, D. et J.P. Ducruc, 1983. Cadre écologique de référence adapté pour l'élaboration des schémas d'aménagement des MRC (guide technique préliminaire). Ministère de l'Environnement du Québec, AME-2, 33 p.

Vieira, Ima Célia G. Arlete Silva de Almeida, Eric A. Davidson, Thomas A. Stone, Cláudio J. Reis de Carvalho et José Benito Guerrero 2003. Classifying successional forests using Landsat spectral properties and ecological characteristics in eastern Amazônia. Remote Sensing of Environment 87 (2003) 470-481.

Wulder Michael A., Joanne C. White, Samuel N. Goward, Jeffrey G. Masek, James R. Irons, Martin Herold, Warren B. Cohen, Thomas R. Loveland et Curtis E. Woodcock 2008. Landsat continuity: Issues and opportunities for land cover monitoring. Remote Sensing of Environment 112 (2008) 955-969.

Wulder, M. A., et Franklin, S. E. 2007. Understanding forest disturbance and spatial pattern: Remote sensing and GIS approaches: CRC Taylor \& Francis.

Wulder, M., Loubier, E., et Richardson, D. 2002. A Landsat-7 ETM+ orthoimage coverage of Canada. Canadian Journal of Remote Sensing, 28(5), 667-671.

Xiao, X., Boles, S., Liu, J., Zhuang, D., et Liu, M. 2002. Characterization of forest types in Northeastern China, using multi-temporal SPOT-4 VEGETATION sensor data. Remote Sensing of Environment, 82(23), 335-348. 